\title{
RENDIMIENTO DE LA MEMORIA PROSPECTIVA EN PERSONAS MAYORES, ADULTOS YJÓVENES
}

\author{
Rúber S. Vélez Larrota ${ }^{1}$, Inmaculada Menacho Jiménez², Esperanza Marchena Consejero², \\ José I. Navarro Guzmán²*, Manuel Aguilar Villagrán² \\ ${ }^{1}$ Universidad de Pamplona - Colombia, ${ }^{2}$ Universidad de Cádiz - España
}

Recibido, septiembre 25/2013

Concepto de evaluación, abril 29/2014

Aceptado, junio 30/2014

\begin{abstract}
Referencia: Vélez Larrota, R. S., Menacho Jiménez, I., Marchena Consejero, E., Navarro Guzmán, J. I. \& Aguilar Villagrán, M. (2014). Rendimiento de la memoria prospectiva en personas mayores, adultos y jóvenes. Acta Colombiana de Psicología, 17 (2), pp. 143-151. DOI:10.14718/ACP.2014.17.2.15
\end{abstract}

Resumen

La finalidad del presente trabajo fue comprobar las diferencias que pudieran existir en Memoria Prospectiva (MP) entre jóvenes, adultos y personas mayores; analizar las relaciones existentes entre variables de comprensión y fluidez verbal y la MP; y verificar la existencia de relaciones entre la percepción subjetiva del estado de salud y el rendimiento de la MP. A través de un diseño transversal, se trabajó con 270 participantes distribuidos en tres grupos de edad: jóvenes de 18 a 28 años; adultos de 45 a 55, y personas mayores de 60 a 80 . Se evaluó a los participantes en relación con sus aptitudes de comprensión y fluidez verbal, así como en la percepción subjetiva de su estado de salud. Posteriormente se realizó un experimento donde se les presentaron párrafos de tres frases en la pantalla del computador y ellos tenían que reconocer palabras previamente acordadas, que indicarían su nivel de MP. Los resultados permitieron confirmar diferencias significativas en memoria prospectiva entre mayores y jóvenes. Sin embargo, no se encontraron diferencias entre jóvenes y adultos. Se detectó la importancia de las aptitudes verbales y la influencia de la percepción subjetiva del estado de salud para tener un mejor rendimiento en estas tareas de laboratorio de MP.

Palabras clave: memoria prospectiva, diferencias de edad, aptitudes verbales, estado salud.

\section{PROSPECTIVE MEMORY PERFORMANCE IN OLDER PEOPLE, ADULTS AND YOUTH}

\begin{abstract}
The aims of this study were to verify the potential differences in prospective memory (PM) among young people, adults and the elderly; analyze the relationships between variables of comprehension and verbal fluency and PM; and finally, verify the existence of a relationship between self-reported health status and performance on PM. A cross- sectional design was used. The study involved 270 participants divided into three age groups: young people aged 18 to 28 years; adults 45 to 55, and seniors 60 to 80 . Their comprehension and verbal fluency skills were assessed as well as their self-perceived health status. Subsequently, an experiment was carried out where participants were presented with paragraphs of three sentences on a computer screen and they had to recognize previously agreed words that would indicate their level of MP. The results established significant differences in prospective memory between adults and older people and between young people and the elderly. But no differences between youth and adults were found..The importance of verbal comprehension and verbal fluency in solving prospective memory experimental tasks was also significant. In addition, a better self-perception of well-being was linked to a higher performance in PM.

Key words: prospective memory, age differences, verbal fluency, well-being.
\end{abstract}

\footnotetext{
* Departamento de Psicología. Universidad de Cádiz, 11510 Puerto Real- Cádiz (España). jose.navarro@uca.es Este trabajo fue posible gracias a la financiación del grupo de investigación HUM634 de la Junta de Andalucía y al contrato OTRI de este grupo con la Diputación provincial de Cádiz. El primer autor fue becario AECID en el Departamento de Psicología de la UCA durante la
} realización del estudio. 


\title{
RENDIMENTO DA MEMÓRIA PROSPECTIVA EM IDOSOS, ADULTOS E JOVENS
}

Resumo

\begin{abstract}
A finalidade do presente trabalho foi comprovar as diferenças que puderam existir na Memória Prospectiva (MP) entre jovens, adultos e idosos; analisar as relações existentes entre variáveis de compreensão e fluência verbal e a MP; e verificar a existência de relações entre a percepção subjetiva do estado de saúde e o rendimento da MP. Através de um desenho transversal, se trabalhou com 270 participantes distribuídos em três grupos de idade: jovens de 18 a 28 anos; adultos de 45 a 55, e idosos de 60 a 80. Avaliaram-se os participantes em relação com suas aptidões de compreensão e fluência verbal, assim como na percepção subjetiva de seu estado de saúde. Posteriormente realizou-se um experimento onde foram apresentados parágrafos de três frases na tela do computador e eles tinham que reconhecer palavras previamente lembradas, que indicariam seu nível de MP. Los resultados permitiram confirmar diferencias significativas em memória prospectiva entre idosos e jovens. Porém, não se encontraram diferenças entre jovens e adultos. Detectou-se a importância das aptidões verbais e a influencia de a percepção subjetiva do estado de saúde para ter um melhor rendimento nestas tarefas de laboratório de MP.

Palavras chave: memoria prospectiva, diferenças de idade, aptidões verbais, estado saúde
\end{abstract}

\section{INTRODUCCIÓN}

La memoria prospectiva (MP) consiste en recordar una acción intencionada que se hará en el futuro. Es la habilidad para recordar una acción que tenemos planeada hacer y que ejecutaremos en algún momento. Ejemplos característicos de memoria prospectiva pueden ser, acordarse de hacer una llamada importante, recordar contestar un email, dar un mensaje a un colega, tomarse un medicamento, o recordar asistir a la cita que teníamos programada con el dentista. Hablar de memoria prospectiva es hablar de intenciones, y las intenciones no siempre pueden llevarse a cabo en el momento en que se forman, por lo que el recuerdo prospectivo exitoso exige que el recuerdo de la intención que debemos realizar se produzca en el momento adecuado en que debe llevarse a cabo (Kvavilashvili \& Fisher, 2007). Estudios recientes ponen especial énfasis en el aspecto social de la memoria prospectiva, dada la repercusión que pueden tener en la vida de las personas (Altgassen, Kliegel, Brandimonte, \& Filippello, 2010). Apagar el gas, cerrar la puerta o el grifo del agua, quedar con alguien, son recuerdos basados en la memoria prospectiva cuyo mal funcionamiento dificulta poder hacer una vida independiente. En ocasiones, las limitaciones evidentes en memoria prospectiva que presentan las personas en los inicios de algún tipo de demencia, determinan su dependencia de terceras personas, dado el riesgo que pueden correr para su salud (Schulz \& Martire, 2004).

Los resultados en tareas prospectivas de tipo verbal, aritmético o de reconocimiento de imágenes -entre otras-, han mostrado, en algunas ocasiones, que las personas mayores tienen una mejor ejecución con respecto a los jóvenes (Cahill, 2006). Sin embargo, este mejor cumplimiento se ha relacionado no sólo con los mecanismos implicados en la MP (tales como la monitorización de información, o los de tipo atencional, inhibición de la información irrelevante y shifting), sino también con la motivación para cumplir las demandas de la tarea, así como con el uso de pistas adecuadas para su cumplimiento (calendarios, anotaciones, etc.). En las tareas de memoria prospectiva ligadas al tiempo, parece que las personas mayores tienen en cambio más problemas para su cumplimiento (Kvavilashvili, Kornbrot, Mash, Cockburn, \& Milne, 2009). En general, por tanto, se ha señalado que los problemas de memoria prospectiva aparecen en las personas mayores cuando se basan únicamente en pistas internas, mientras que cuando existen pistas externas, tanto debidas a la propia tarea, como al uso de dispositivos (agendas, calendarios, alarmas, etc.), los resultados de las personas mayores pueden llegar a ser incluso mejores que los de los jóvenes.

Pero la investigación no es concluyente (Kliegel, Martin, McDaniel, Einstein, \& Moor, 2007; Henry, Macleod, Philips, \& Crawford, 2004). La complejidad de la MP, debido a la existencia de múltiples procesos implicados en ella, junto a resultados contradictorios en su declive asociado a la edad (Serrani, 2010; Wang, Dew, \& Giovanello, 2010), hacen que el estudio de las características del recuerdo prospectivo de jóvenes, adultos y personas mayores, sea un tema de especial interés. Existen dos procesos que pudieran estar relacionados con un rendimiento adecuado en MP. Se trata del dominio de los componentes verbales que tiene el sujeto y de los factores motivacionales relacionados con la salud, especialmente en personas mayores. Aunque existen trabajos bien fundamentados sobre la importancia del tipo de tareas en la eficiencia de la MP, se ha encontrado que aquellas que requieren una alta demanda cognitiva tienen menos probabilidad de ser recordadas (McDaniel, Howard, $\&$ Butler, 2008). Sin embargo, hay menos evidencias sobre 
el papel de la fluidez verbal y de la comprensión verbal en la memoria prospectiva. En ocasiones han sido las tareas experimentales de tipo verbal las que se han utilizado para evaluar el rendimiento en MP, diferenciándose los resultados en función de la intensidad de la demanda cognitiva de la tarea, más que por las características de los componentes verbales (McDaniel, \& Scullin, 2010). Por ello entendemos que la fluidez y la comprensión verbal pueden tener un papel significativo en la MP, por cuanto permitirían una mayor discriminación entre los componentes verbales de las tareas. Y en relación con los aspectos motivacionales, se ha sugerido que los estados de salud reales o subjetivos juegan un importante papel en el rendimiento cognitivo (Baker, Gazmararian, Sudano, \& Patterson, 2000), entendiendo que una percepción subjetiva deficiente sobre el estado de salud puede limitar las expectativas de éxito en tareas cognitivas (Menacho, 2008).

La finalidad del presente trabajo tuvo tres puntos de interés. Por un lado, comprobar las diferencias que pudieran existir en MP entre tres grupos de edad bien diferenciados: jóvenes, adultos y mayores. Dado que la literatura existente sobre la psicología evolutiva de la MP no es concluyente, se planteó la hipótesis de que los resultados en MP no son función de la edad. De ser así, habría sido necesario relacionar las dificultades de memoria en las personas mayores con la disminución de los recursos atencionales y con los mecanismos intencionales (existentes en la memoria prospectiva), en la línea de la teoría preparatoria y atencional de la memoria (PAM) de Smith, Hunt, McVay, \& McConnell (2007). Esta teoría señala que un recuerdo intencional puede ser recuperado sólo si el individuo se involucra en un proceso preparatorio de aquellos estímulos que nos señalan que tenemos que realizar una determinada tarea. Es decir, recordaríamos una cita futura con un amigo, si previamente realizamos un monitoreo de dicha cita cuando la estamos almacenando en la memoria. Se trata de un campo que necesita una mayor exploración experimental, no sólo por el conocimiento de las diferencias evolutivas sino porque permitiría también desarrollar medidas preventivas, en su caso. El segundo interés de este trabajo fue analizar las relaciones existentes entre variables de comprensión y fluidez verbal y la MP. El hecho de que las tareas del experimento diseñado estuvieran basadas en contenidos escritos que los participantes debían leer, justificó la evaluación previa de estos dos factores verbales. Nuestra hipótesis fue establecer una relación positiva entre el nivel de fluidez y comprensión verbal y el rendimiento en MP. Se consideró que un mayor nivel de fluidez y comprensión verbal incrementaría el rendimiento en tareas de memoria prospectiva, liberando más recursos cognitivos, al permitir una mejor focalización de la atención en los contenidos de la tarea. Finalmente, dado que la percepción de la salud de las personas mayores es un factor que puede tener decidida influencia en las expectativas de rendimiento cognitivo, se planteó una tercera hipótesis de trabajo en la que se pretendía verificar la existencia de una relación entre la percepción subjetiva sobre el estado de salud y el rendimiento en tareas de MP. En este sentido, una mejor autopercepción de la salud haría más probable unos resultados favorables en tareas de memoria prospectiva, puesto que generaría un mayor nivel de autoconfianza en sus recursos metacognitivos.

\section{MÉTODO}

\section{Participantes}

La muestra estaba formada por un total de 270 hombres y mujeres pertenecientes a varios núcleos urbanos de más de 50 mil habitantes, con edades comprendidas entre los 18 y 80 años. En general, procedían de un estrato socioeconómico medio y medio-bajo. El $60 \%$ de los participantes eran mujeres. E1 34.4\% de la muestra trabajaba, un 65.6\% no lo hacía, o eran estudiantes; el 10\% de los participantes tenía estudios universitarios, el $36.7 \%$, estudios medios, un $6,3 \%$, estudios secundarios, y un $18,9 \%$, estudios de primaria; el $18,1 \%$ no tenía estudios.

La muestra fue elegida según su grado de disponibilidad y accesibilidad, y se dividió en tres grupos de edad de 90 sujetos cada uno. La población joven (18 a 28 años) procedía del primer curso de las titulaciones universitarias de maestros que participaban voluntariamente en el estudio como parte de su formación práctica de una de sus asignaturas. En cuanto a la población adulta (45 a 55 años), eran participantes voluntarios de varios centros sociales de ocio de adultos, ubicados en los barrios urbanos donde se organizó esta investigación como una labor educativa complementaria a su programa de actividades. Se seleccionaron adultos sanos, con diferentes niveles educativos y socioculturales, que no presentaban índices de deterioro cognitivo posterior al juicio clínico del evaluador. Por último, la población mayor $(60 \mathrm{a}$ 80 años) eran personas del mismo centro de adultos mencionado anteriormente, que formaban parte del alumnado del aula universitaria de mayores. Se trataba de personas mayores pero que no tenían diagnósticos de demencia o enfermedades graves.

\section{Instrumentos}

Perfil de Salud de Nottingham (PSN). Se utilizó la adaptación española de Antó, Alonso, \& Moreno (1990). Se trata de un cuestionario de 45 ítems que mide el estado de salud general, en las categorías de energía, dolor, reacción emocional, sueño, aislamiento social y movilidad física. Se obtiene cero puntos cuando la persona contesta negativamente a todos los ítems de la dimensión y no padece ningún 
problema de los que ésta refleja. La puntuación máxima es 45 e indica una percepción subjetiva de salud negativa en todos los aspectos que contempla el cuestionario. Los valores de fiabilidad para el PSN calculados con el alfa de Cronbach fueron de.72.

Test de Aptitudes Mentales Primarias (PMA). Se utilizó la versión española adaptada por Secadas (1988). Se trata de una prueba bien conocida en la evaluación de aptitudes intelectuales. La batería consta de cinco pruebas que detectan aisladamente cinco factores: comprensión verbal, espacial, razonamiento, cálculo numérico y fluidez verbal. La duración de la prueba completa es de 26 minutos aproximadamente. La edad de aplicación es de 10 años en adelante. Utilizamos para este estudio solamente los factores de comprensión verbal (V) y fluidez verbal (F). La fiabilidad arroja un alfa de Cronbach $=.93$

\section{Procedimiento}

El estudio se desarrolló a lo largo de tres momentos distintos. En primer lugar, los participantes fueron citados por los investigadores e informados acerca del proyecto de la Universidad de Cádiz sobre el desarrollo de la memoria. Todos recibieron una ficha informativa que recopilaba datos acerca de las siguientes variables: edad, ocupación, género, nivel de estudios y estado civil. Por último, se aplicó el cuestionario PSN. Estas evaluaciones fueron individuales para los adultos y personas mayores, o en pequeños grupos de cinco a siete para los alumnos universitarios, y tenían una duración aproximada de 20 a 30 minutos.

En segundo lugar, se volvió a citar a los participantes para recibir la aplicación de dos factores de la batería PMA: comprensión verbal (V) y fluidez verbal (F). Se tenía interés por conocer su relación con la edad, y a su vez, con los resultados del experimento de memoria prospectiva. Para el factor de comprensión verbal, los participantes recibían las instrucciones por parte del experimentador a través de un ejemplo incluido en la prueba. Se les dictaba de la misma forma, los participantes respondían, y el investigador finalmente daba la respuesta correcta. Una vez realizado el ejemplo, se les dijo que disponían de cuatro minutos para realizar toda la prueba, tal como sugiere el manual de administración del test. Igualmente, se procedió con la prueba del factor fluidez verbal (F), la cual fue explicada por el experimentador siguiendo las normas de aplicación. Los participantes recibían las instrucciones por parte del experimentador a través de un ejemplo donde se les daba la solución. Finalizado el ejemplo del test, y una vez se tenía seguridad de que se había entendido la tarea, los participantes disponían de cinco minutos para escribir todas las palabras que se les ocurrieran, sin preocuparse por los errores ortográficos. La duración aproximada de la segunda sesión fue de entre 20 y 30 minutos.

Las dos primeras sesiones se llevaron a cabo en aulas universitarias, en el caso de los jóvenes y mayores, y en salas de centros culturales en el caso de los adultos; ambos recintos estaban debidamente acondicionados y aislados de posibles ruidos distractores. Se realizaron en buenas condiciones de comodidad y concentración. En todos los casos, las pruebas fueron administradas por los autores de este estudio.

En tercer lugar, los participantes acudieron a la sesión del experimento sobre MP diseñado a través del software Superlab 3.0, con base en los trabajos de McDaniel, Einstein, Stout, \& Morgan (2003). Dicho experimento se encontraba disponible en cinco ordenadores de una sala de informática amplia y confortable, lo cual permitió que se pudiera realizar individualmente por cinco personas al mismo tiempo. Sin embargo, para la población mayor, el experimento se realizó en la mayoría de los casos de forma individual, con una computadora portátil, debido a las numerosas preguntas que hacían algunos de ellos y que podían influir o distraer a los demás.

Una vez que los participantes estaban delante de la computadora, el programa les proporcionaba un párrafo de instrucciones donde se leía lo siguiente: "Con esta prueba, estamos investigando su capacidad para acordarse de realizar una acción en el futuro. Así, cada vez que vea la palabra "técnica" o la palabra "sistema" en el párrafo que aparecerá en la pantalla, debe presionar la barra espaciadora. Puede hacer esto tan pronto como vea la palabra "técnica" o "sistema", incluso si tiene que interrumpir lo que está haciendo." Otras instrucciones específicas a los participantes fueron: "La prueba que vamos a realizar a continuación, es parte de un procedimiento diseñado para evaluar nuestra capacidad de memoria. En concreto, la habilidad para recordar una acción que tenemos planeada hacer y que ejecutaremos en algún momento en el futuro, mientras realizamos otras actividades de la vida diaria. Para llevar a cabo estas tareas, no necesita saber nada específico acerca de cómo funciona una computadora. Si tiene alguna pregunta, la persona encargada de administrar la prueba se la responderá. Por favor, lea atentamente los párrafos que aparecen en la pantalla y si está de acuerdo, puede dar su consentimiento para participar en esta prueba. Cuando esté listo para empezar, presione la tecla avance para continuar". Por último, el programa facilitaba las instrucciones para comprender lo que debían hacer cada vez que leyeran los párrafos: “...se presentará una serie de párrafos que podrá leer durante un tiempo aproximado de 60 segundos. A continuación, se le darán un par de tareas que tendrá que 
realizar antes de contestar a las preguntas de comprensión de los párrafos leídos anteriormente".

Antes de comenzar con la presentación de los 20 párrafos y sus tareas correspondientes, los participantes realizaban un ejemplo para facilitar la correcta comprensión del procedimiento. Para una mayor precisión, se colocó un rótulo en el teclado del computador con las palabras barra espaciadora y avance escritas en negrita, evitando así que presionaran una tecla diferente y que dificultase el registro correcto de los resultados del experimento.

Los párrafos eran de cultura muy general sobre Historia, Geografía, Sociedad, etc., y estaban compuestos por tres oraciones cada uno. Tras cada párrafo, los participantes debían contestar a dos tareas de sinónimos, dos tareas de preguntas sencillas, y dos tareas de preguntas de comprensión sobre el párrafo. Para contestar las tareas, tenían que pulsar un número del 1 al 4 en el teclado del ordenador, el cual correspondía a la opción elegida por el participante, pues las preguntas eran de opción múltiple.

La MP estuvo representada en el experimento en siete de los 20 párrafos. Es decir, en los párrafos 3, 5, 8, 10, 15,18 y 20 aparecía, de forma inadvertida para los participantes, alguna de las palabras clave (técnica o sistema) ante la cual los participantes debían presionar la barra espaciadora tal como se había dicho en las instrucciones, indicando así que recordaban las palabras que previamente se les habían indicado. Esta sería la medida de la MP. El tiempo total empleado en responder la prueba variaba de 30 minutos en los jóvenes, a 60 minutos en los participantes de mayor edad, contabilizando la presentación de los experimentadores, las explicaciones del experimento y los descansos. Para el registro de los aciertos en las palabras que medían la MP se establecieron tres categorías: a) $0,1,2$. Los participantes que obtuvieron 0-1-2 aciertos se codificaron con 0 ; b) 3-4-5 aciertos, con un 1 ; y c) 6-7 aciertos, con un 2. Otra variable que se tuvo en cuenta en el experimento fue el tiempo empleado en cada uno de los aciertos en MP. Igualmente, en el análisis de resultados se estableció el número de errores en MP y el tiempo que los participantes tardaban en dar la respuesta errónea. Todos los datos eran registrados de forma automática de acuerdo con el diseño del experimento realizado con Superlab 3.0. Una vez completados, fueron cargados a la aplicación informática SPSS.13 para su tratamiento estadístico.

\section{Consideraciones éticas}

Al iniciar el estudio, se explicó el objetivo de la investigación y se dio la libertad de decidir si participaban o no en el estudio. Se consiguió el consentimiento informado de todos los participantes, guardando la confidencialidad y el anonimato en los datos.

\section{RESULTADOS}

Lo que se esperaba con esta investigación era determinar si existían, o no, diferencias significativas en la MP de la muestra de participantes de 18 a 80 años de edad. Los resultados se presentan teniendo en cuenta, en primer lugar, las medidas de MP en relación con la edad. Y posteriormente, los resultados en relación con las medidas de comprensión y fluidez verbal, además de los datos obtenidos con respecto a la percepción subjetiva del estado de salud.

La media de aciertos en el grupo de jóvenes (18-28 años) fue la más alta $(M=1.93 ; d t=.292)$, lo cual indica que su MP fue mejor que la de los adultos (45-55 años) $(M=1.89$; $d t=.350)$ y mucho mejor que la MP del grupo de mayores (60-80 años), $(M=1.33 ; d t=.734)$. El grupo de jóvenes obtuvo una media inferior en el tiempo medio de aciertos $(M=26.19 ; d t=8.721)$, indicando que se demoraba menos cuando acertaba, en comparación con los otros grupos de edad de los adultos $(M=29.98 ; d t=9.157)$ y los mayores. Estos eran los que tardaban más cuando acertaban $(M=35.64$; $d t=19.38)$. Cuando los jóvenes cometían errores, tardaban más en dar su respuesta $(M=6.89 ; d t=1.66)$ que el grupo de los adultos $(M=5.98 ; d t=1.44)$. Los mayores, cuando daban una respuesta errónea, fueron el grupo de edad que más tardaba en hacerlo $(M=24.85 ; d t=2.05)$.

Una vez realizados los contrastes de medias se observó la existencia de diferencias significativas entre los grupos en cada una de las medidas experimentales: aciertos $(F(2 g l)=43.77 ; p<.0001)$, errores, tiempo medio para los aciertos $(F(2 g l)=11.40 ; p<.0001)$, y tiempo medio para los errores $(F(2 g l)=33.65 ; p<.0001)$.

Se contrastó la hipótesis de igualdad de varianza con la prueba de Levene y la prueba $t$ de Student para la comparación de medias (ver Tabla 1). Se encontraron diferencias significativas entre los grupos de edad. En efecto, existían diferencias significativas en los aciertos entre los grupos de mayores versus de jóvenes, y entre los adultos versus los mayores. El tiempo medio de errores entre el grupo de los mayores y el grupo de jóvenes fue también significativo. Cuando se compararon adultos y mayores, el desempeño fue mejor en la población de adultos que en la población mayor. Sin embargo, las comparaciones entre jóvenes versus adultos no mostraron diferencias estadísticamente significativas. Mientras que las comparaciones entre los grupos de jóvenes versus mayores y entre los grupos de adultos versus mayores fueron siempre significativas. No fue así para las comparaciones jóvenes versus adultos, para ninguna de las medidas de la MP.

En relación con los resultados descriptivos obtenidos por cada uno de los grupos de edad en los valores de comprensión verbal, se mencionan las diferentes medias dependiendo de 
Tabla 1.

Resultados de la prueba de Levene $(F)$ para muestras independientes y de la prueba $t$ de Student para la comparación de medias entre los grupos de edad para diversas medidas de Memoria Prospectiva (MP).

\begin{tabular}{|c|c|c|c|c|c|c|c|c|c|}
\hline & \multicolumn{3}{|c|}{ Jóvenes-Mayores } & \multicolumn{3}{|c|}{ Jóvenes-Adultos } & \multicolumn{3}{|c|}{ Adultos-Mayores } \\
\hline & $F$ & $t^{1}$ & $\begin{array}{c}\text { Dif } \\
\text { Medias } \\
\end{array}$ & $F$ & $t^{1}$ & $\begin{array}{c}\text { Dif } \\
\text { Medias } \\
\end{array}$ & $F$ & $t^{1}$ & $\begin{array}{c}\text { Dif } \\
\text { Medias } \\
\end{array}$ \\
\hline MP Aciertos & $59.74^{* *}$ & $7.05^{* *}$ & -48.04 & .05 & -0.2 & -.03 & $70.53^{* *}$ & $7.37^{* *}$ & 1.96 \\
\hline MP Errores & $59.74 * *$ & $7.06^{* *}$ & 1.92 & .05 & .2 & .03 & $70.53^{* *}$ & $-7.37^{* *}$ & -1.96 \\
\hline $\begin{array}{l}\text { Tiempo reacción } \\
\text { Aciertos }\end{array}$ & $40.05^{* *}$ & $-7.06^{* *}$ & -1.92 & .47 & -2.84 & -3786.8 & $35.2 * *$ & $-2.51^{* *}$ & -5663.5 \\
\hline $\begin{array}{l}\text { Tiempo reacción } \\
\text { Errores }\end{array}$ & $9.88^{* *}$ & $-4.22^{* *}$ & -4950.3 & .51 & .39 & -911.3 & $17.05^{* *}$ & $-7.12^{* *}$ & 18869.3 \\
\hline
\end{tabular}

(1) $=178 \mathrm{gl} ;\left(^{* *}\right)=p<.0001$

la edad de los grupos participantes. El grupo de jóvenes (1828 años) obtuvo una media superior al resto de los grupos en la prueba de comprensión verbal $(M=30.44 ; d t=7.54)$. Asimismo, el grupo con menor puntuación corresponde a las personas mayores (60-80 años), $(M=13.98 ; d t=10.00)$. Los adultos (45-55 años) obtuvieron una media inferior a la de los jóvenes $(M=28.70 ; d t=12.15)$, pero por encima de los mayores. Un resultado similar se presenta en la prueba de fluidez verbal (jóvenes: $M=43.67 ; d t=11.17$; adultos: $M=32.16 ; d t=8.63$; mayores: $M=27.034 ; d t=11.24)$. En cuanto a los resultados con el Perfil de Salud de Nottingham, el grupo de mayores (60-80 años) obtuvo una media superior al resto de los grupos (mayores: $M=9,83 ; d t=7,56$; jóvenes: $M=2,8 ; d t=3,2$; adultos: $M=5,6 ; d t=6,6$ ), lo que permite percibir la existencia de un mayor deterioro subjetivo de salud en las personas de más edad.
Otro análisis comparativo consistió en correlacionar la variable edad con la ejecución de las tareas de MP según el experimento diseñado y las medidas de comprensión y fluidez verbal y percepción subjetiva del estado de salud (ver Tabla 2). En efecto, se correlacionaron tanto las puntuaciones directas del factor comprensión verbal, fluidez verbal y el PSN, como el total de aciertos, tiempo medio de aciertos, tiempo medio de errores en MP, con la edad de los participantes. Los valores encontrados indicaron que a mayor edad, la puntuación en el factor comprensión verbal $(r=-0.52 ; p<.0001)$ y fluidez verbal $(r=-.55 ; p<.001)$ fue menor. El PSN empeoraba con la edad $(r=.42 ; p<.001)$. Y a mayor edad, se cometieron más errores en $\mathrm{MP}(r=.407$; $p<.01)$ y también se empleaba más tiempo para acertar en las diversas medidas de MP.

Tabla 2.

Correlaciones obtenidas entre las puntuaciones en comprensión verbal, fluidez verbal Perfil de Salud de Nottingham, las diversas medidas de memoria prospectiva (MP) y la edad de los participantes.

\begin{tabular}{lcccccccc}
\hline & $\mathbf{V}$ & $\mathbf{F}$ & $\mathbf{N}$ & $\mathbf{M P a}$ & $\mathbf{M P e}$ & $\mathbf{T M a}$ & TMe & Edad \\
\hline $\mathbf{V}$ & 1 & & & & & & & \\
$\mathbf{F}$ & $0.57^{* *}$ & 1 & & & & & & \\
$\mathbf{N}$ & $0.44^{* *}$ & $0.36^{*}$ & 1 & & & & & \\
$\mathbf{M P a}$ & $0.47^{* *}$ & $0.32^{*}$ & $-0.33^{*}$ & 1 & & & & \\
$\mathbf{M P e}$ & $-0.47^{* *}$ & $-0.32^{*}$ & $0.33^{*}$ & -1.00 & 1 & & & \\
$\mathbf{T M a}$ & $-0.34^{*}$ & $-0.26^{*}$ & 0.15 & 0.08 & -0.08 & 1 & & \\
$\mathbf{T M e}$ & $-0.42^{* *}$ & $-0.27^{*}$ & $0.27^{*}$ & $-0.65^{* *}$ & $0.65^{* *}$ & 0.03 & 1 & \\
$\mathbf{E d a d}$ & $-0.52^{* *}$ & $-0.55^{* *}$ & $0.42^{* *}$ & $-0.40^{* *}$ & $0.40^{* *}$ & $0.28^{*}$ & $0.35^{*}$ & 1 \\
\hline
\end{tabular}

Nota. V: Comprensión Verbal, F: Fluidez Verbal, N: Perfil de Salud de Nottingham, MPa: Aciertos en Memoria Prospectiva, MPe: Errores Memoria Prospectiva, TMa: Tiempo Medio para los aciertos, TMe: Tiempo Medio para los errores.

$\left({ }^{* *}\right) \mathrm{p}<0.001 ;\left(^{*}\right) \mathrm{p}<0.01$. 
Tabla 3.

Regresión jerárquica realizada en relación al nivel de aciertos en memoria prospectiva (MP).

\begin{tabular}{lllcccc}
\hline Modelo & Medidas de MP & \multicolumn{1}{c}{ Variables } & $\boldsymbol{r}$ & $\boldsymbol{r}^{2}$ corregido & $\boldsymbol{F}$ & Sig. \\
\hline 1 & Aciertos en MP & Edad & .311 & .136 & 22.418 & .000 \\
2 & & Perfil de Salud de Nottingham & .423 & .223 & 19.59 & .000 \\
3 & & Comprensión verbal & .496 & .270 & 16.15 & .001 \\
\hline
\end{tabular}

Tras realizar las correlaciones entre las diferentes medidas de memoria prospectiva, se calculó una regresión jerárquica con la intención de conocer el modelo que explicaba la mayor cantidad de varianza. Como puede verse en la tabla 3, el modelo que resultó significativo estuvo compuesto por la variable edad, que explicaba el $13.6 \%$ de la varianza; seguido por el estado de salud, evaluado a través del cuestionario de Nottingham, el cual añadía hasta el $22 \%$ de la varianza explicada; y finalmente, la comprensión verbal que explicaba hasta el $27 \%$ de la misma. De esta manera, el tipo de tarea realizada en el experimento correlacionaba con los componentes verbales de la misma. Asimismo, la edad constituía un factor importante en la pérdida de memoria prospectiva.

\section{DISCUSIÓN}

En este trabajo se planteó como objetivo general estudiar las diferencias en memoria prospectiva en jóvenes, adultos y personas mayores, valorando la capacidad de cada grupo de estudio para recordar algo que tendrá que realizar en un futuro inmediato. Asimismo, se quería comprobar la relación que existe entre variables como la comprensión y la fluidez verbal con el rendimiento en tareas de MP en un contexto de laboratorio. Finalmente, se pretendía saber también cuál es la influencia que tiene la percepción subjetiva del estado de salud en la MP.

Los participantes más jóvenes (18-25 años) obtuvieron los mejores resultados en las tareas de MP. Tardaron menos tiempo en responder con aciertos, pero no fueron los mejores en cuanto al tiempo empleado para responder con errores. Los participantes de mayor edad (60-80 años) no sólo fueron más lentos que todos los demás grupos en la velocidad de respuesta para encontrar sus aciertos y errores, sino que tuvieron menos aciertos y más errores que los demás a la hora de localizar las palabras claves que nos indicaban su MP.

Estos resultados vienen a corroborar un menor rendimiento en tareas de MP para los mayores de 60 años (Kvavilashvili, et al., 2009). Sin embargo, no confirman necesariamente el declive de la MP debido a la edad, tal como señalábamos en nuestra hipótesis inicial. Los participantes más jóvenes aciertan significativamente con más palabras claves que los ancianos, pero no es así cuando los comparamos con el grupo de mediana edad (45-55 años). Mientras que las comparaciones entre los grupos de jóvenes versus mayores y entre los grupos de adultos versus mayores fueron siempre significativas. No fue así para las comparaciones jóvenes versus adultos, para ninguna de las medidas de la MP. Una posible explicación del menor rendimiento del grupo de mayores puede estar relacionada con el hecho de que en contextos de tareas de laboratorio, como el presentado en este estudio, hay una clara desventaja para los participantes de mayor edad (Bailey, Henry, Rendell, Phillips, \& Kliegel, 2010). Mientras que cuando la tarea tiene una clave emocional para facilitar el recuerdo en memoria prospectiva, las diferencias en la ejecución de adultos mayores y adultos jóvenes se reducen. Sin embargo, cuando la clave que se ofrece es neutral, es decir, su valor no es ni positivo ni negativo, se incrementan las diferencias en memoria prospectiva, siendo menor el rendimiento de los adultos mayores (Altgassen, Phillips, Henry, Rendell, \& Kliegel, 2010).

También fue interesante poder interpretar la ausencia de diferencias significativas entre los grupos de jóvenes y adultos en MP en relación con los contrastes en el uso de estrategias eficientes. En la expectativa teórica del modelo del deterioro del hardware debido a la edad (Graciani, Banegas, Guallar, Domínguez, \& Rodríguez, 2006; Millán, Tubío, Pita, González, Lorenzo, \& Maseda, 2009) se plantea la mejora en el manejo de estrategias más efectivas para recordar. En este sentido, las personas más maduras habrían tenido mayor experiencia para comprobar cuáles de sus estrategias de recuerdo les han sido más efectivas. Y esto es particularmente interesante dado que los participantes de menor edad procedían de entornos universitarios, en contraste con los participantes del grupo de edad de 45-55 años.

Otro aspecto tenido en cuenta en nuestro estudio fue la conexión entre la percepción subjetiva que se tiene de la salud y el rendimiento en las tareas de MP. Hay diferentes evidencias que indican la importancia de los aspectos sociales y emocionales de los procesos cognitivos en general 
(Navarro, Alcalde, Marchena, \& Aguilar, 2003; Washburn, \& Sands, 2006), y en particular en la MP (Altgassen, Phi1lips, Henry, Rendell, \& Kliegel, 2010). Los resultados encontrados aquí confirman la hipótesis inicial de que a medida que nos hacemos mayores, percibimos de forma más pesimista nuestro estado de salud. Una peor percepción subjetiva de nuestro estado de salud crea también peores expectativas respecto al rendimiento, lo cual se puede traducir en una actividad mental cada vez menor. Se podría decir que se cumple el efecto Pigmalion (Rosenthal, \& Jacobson, 1992): no me siento bien, no voy a ser capaz de hacerlo bien, y finalmente lo hago mal. En otros trabajos se ha podido comprobar esta tendencia para actividades de atención y memoria a corto y largo plazo. Así, en Navarro et al., $(2003,2009)$ se entrenó a personas mayores de 65 años sanas pero institucionalizadas, en diversas tareas cognitivas. Aquellas que tenían peor percepción subjetiva de su estado de salud, evaluado a través del perfil de salud de Nottingham, solían tener también peor rendimiento en las actividades de rehabilitación cognitiva llevadas a cabo en la institución.

Disponer de un mayor nivel de comprensión verbal mejoraba en la presente muestra los resultados en MP, al igual que ocurría con la fluidez verbal. El hecho de que las tareas del experimento estuvieran basadas en contenidos escritos que los participantes debían leer, justificó la evaluación previa de estos dos factores verbales (McDaniel, et al., 2003). El grupo de los más jóvenes tuvo mejores resultados en comprensión y fluidez verbal y, de nuevo, fueron los mayores de 60 años los que puntuaron peor. Las diferencias encontradas entre los grupos de edad en los parámetros de la MP fueron muy similares a las encontradas en el ámbito de las medidas verbales. Y en la relación establecida entre estas variables verbales y la MP, se encontró que en las tareas experimentales evaluadas, aquellos participantes con menos puntuación en comprensión y fluidez verbal las ejecutaron peor, tanto en respuestas correctas como en tiempos de respuesta para los errores y los aciertos. En este sentido, el trabajo confirma la hipótesis de que un mayor grado de fluidez y comprensión verbal ampliaría el rendimiento en memoria prospectiva, posiblemente al permitir una mejor focalización de la atención en los contenidos de la tarea, liberando más recursos cognitivos.

Pero no todas las dimensiones de la memoria cambian de la misma manera, ni en la misma dirección, lo cual sugiere una multi-direccionalidad y una multi-dimensionalidad de la influencia de la edad en la memoria (Balota, Dolan, \& Duchek, 2000). En el caso de la MP, parece deducirse de este estudio que las actividades de laboratorio donde había un proceso de comprensión verbal, se vieron afectadas por el paso de los años, dado que el grupo de mayores de 60 años fue el que peor las ejecutó, seguido por el grupo de 45-55 años, y finalmente por el grupo de los más jóvenes.

En cualquier caso, la MP puede ser esencial para conservar una adecuada calidad de vida. Aunque existen numerosos dispositivos artificiales que nos pueden avisar cuáles son las actividades que debemos hacer en el futuro, uno de los retos de la futura investigación en MP será diseñar metodologías que permitan un uso estratégico más eficiente de los recursos intelectuales para realizar estas actividades programadas en el pasado. Pese a las dificultades encontradas en algunos estudios a la hora de mejorar el recuerdo prospectivo en personas mayores o muy mayores (Schnitzspahn, \& Kliegel, 2009; McDaniel \& Scullin, 2010), es posible mejorar las funciones cognitivas en personas con deterioro debido a la edad (Menacho, 2008). Y este hecho es de especial relevancia si tenemos en cuenta que este deterioro implícito puede ser inicio del desarrollo de algunas formas de demencia a medio plazo (Dechamps, Onifade, Decamps, \& Bourdel-Marchasson, 2009).

Futuros estudios podrían aportar más conocimiento sobre el funcionamiento de la MP en personas mayores, ajustando las tareas de laboratorio a las actividades de la vida cotidiana y comprobando si el uso de estrategias que impliquen un manejo eficiente de la comprensión y fluidez verbal puede paliar la pérdida de MP en personas mayores.

\section{REFERENCIAS}

Antó, J. M., Alonso, J., \& Moreno, C. (1990). Spanish version of the Nottingham health profile: Translation and preliminary validity. American Journal of Public Health, 80, 704708.

Altgassen, M Kliegel., , M., Brandimonte, M., \& Filippello, P. (2010). Are older adults more social than younger adults? Social importance increases older adults' prospective memory performance. Neuropsychology, Development, and Cognition. Section B, Aging, Neuropsychology and Cognition, 17, 312-28. Doi: 10.1080/13825580903281308

Altgassen, M., Phillips, L., Henry, J. D., Rendell, P. G., \& Kliegel, M. (2010). Emotional target cues eliminate age differences in prospective memory. The Quarterly Journal of Experimental Psychology, 63, 1057-1064. Doi: 10.1080/17470211003770920.

Bailey, P. E., Henry, J. D., Rendell, P. G., Phillips, L. H., \& Kliegel, M. (2010). Dismantling the "age-prospective memory paradox": The classic laboratory paradigm simulated in a naturalistic setting. The Quarterly Journal of Experimental Psychology, 63, 646-652. Doi: 10.1080/17470210903521797.

Baker, D. W., Gazmararian, J. A., Sudano, J., \& Patterson, M. (2000). The association between age and health literacy 
among elderly persons. Journal of Gerontology: Psychological Sciences, 55, 68-74.

Balota, D. A., Dolan, P. O., \& Duchek, J. M. (2000). Memory changes in healthy older adults. In E. Tulving \& F. I. M. Craik. (Ed.), Handbook of memory. (pp. 395-410). New York: Oxford University Press.

Cahill, A. L. (2006). The effects of ageing, task interruption and planning on prospective memory. Brisbane, Australia: Griffith University.

Dechamps, A.; Onifade, C.; Decamps, A.; Bourdel-Marchasson, I. (2009). Health-related quality of life in frail institutionalized elderly: Effects of a cognition-action intervention and Tai Chi. Journal of Aging and Physical Activity, 17, 236-248.

Graciani, A., Banegas, J., Guallar, P., Domínguez, V., \& Rodríguez, F. (2006). Cognitive assessment of the non-demented elderly community dwellers in Spain. Dementia and Geriatric Cognitive Disorders, 21, 104-112. Doi: 10.1159/000090509.

Henry, J. D., Macleod, M. S., Philips, L. H., \& Crawford, J. R. (2004). A meta-analytic review of prospective memory and aging. Psychology and Aging, 19, 27-39. 10.1037/08827974.19.1.27.

Kliegel, M., Martin, M., McDaniel, M. A., Einstein, G. O., \& Moor, C. (2007). Realizing complex delayed intentions in young and old adults: The role of planning aids. Memory \& Cognition, 35, 1735-1746. Doi: 10.3758/BF03193506.

Kvavilashvili, L., \& Fisher, L. (2007). Is time-based prospective remembering mediated by self-initiated rehearsals? role of incidental cues, ongoing activity, age, and motivation. Journal of Experimental Psychology: General, 136, 112132. Doi: 10.1037/0096-3445.136.1.112.

Kvavilashvili, L., Kornbrot, D. E., Mash, V., Cockburn, J., \& Milne, A. (2009). Differential effects of age on prospective and retrospective memory tasks in young, youngold, and old-old adults. Memory, 17, 180-196. Doi: 10.1080/09658210802194366.

McDaniel, M. A., Einstein, G. O., Stout, A. C., \& Morgan, Z. (2003). Aging and maintaining intentions over delays: Do it or lose it. Psychology and Aging, 18, 823-835. Doi: 0.1037/0882-7974.18.4.823.

McDaniel, M. A., Howard, D. C., \& Butler, K. M. (2008). Implementation intentions facilitate prospective memory under high attention demands. Memory and Cognition, 36, 716-724. Doi: 10.3758/MC.36.4.716

McDaniel, M. A., \& Scullin, M. K. (2010). Implementation intention encoding does not automatize prospective memory responding. Memory \& Cognition, 38, 221-232. Doi: 10.3758/MC.38.2.221v.
Menacho, I. (2008). Validación del programa de modificabilidad cognitiva en población adulta. Tesis doctoral. Cádiz, España: Universidad de Cádiz. https://www.educacion.es/ teseo/mostrarRef.do?ref $=872070$

Millán, J.C., Tubío, J., Pita, S., González, I., Lorenzo, T., \& Maseda, A. (2009). Prevalence of cognitive impairment: Effects of level of education, age, sex and associated factors. Dementia and Geriatric Cognitive Disorders, 28, 455460. Doi: $10.1159 / 000257086$.

Navarro, J.I., Alcalde, C., Marchena, E., \& Aguilar, M. (2003). Programa para la mejora de la atención en personas mayores. Psicología Conductual, 11, 179-186.

Navarro, J.I., Menacho, I., Alcalde, C., Marchena, E., Ruiz, G., \& Aguilar, M. (2009). Cognitive changes among institutionalized elderly people. Educational Gerontology, 35, 1-18. Doi: $10.1080 / 03601270802608568$.

Rosenthal, R. \& Jacobson, L. (1992). Pygmalion in the Classroom: Teacher Expectation and Pupils' Intellectual Development. New York, USA: Irvington Publishers.

Secadas, J.L. (1988). Test de Aptitudes Mentales Primarias. Madrid: TEA.

Serrani, D. (2010). Memoria cotidiana en población de adultos mayores: un estudio longitudinal de 10 años. Acta Colombiana de Psicología, 13(2), 91-105.

Schnitzspahn, K. M. \& Kliegel, M. (2009). Age effects in prospective memory performance within older adults: The paradoxical impact of implementation intentions. European Journal of Ageing, 6, 147-155. Doi: 10.1007/s10433-0090116-x.

Schulz, R. \& Martire, L. M. (2004). Family caregiving of persons with dementia: prevalence, health effects, and support strategies. The American Journal of Geriatric Psychiatry, 12, 240-249.

Smith, R. E., Hunt, R. R., McVay, J. C., \& McConnell, M. D. (2007). The cost of event-based prospective memory: Salient target events. Journal of Experimental Psychology: Learning, Memory, and Cognition, 33, 734-746.

Wang, W., Dew, I. T. Z., \& S. Giovanello, K. (2010). Effects of aging and prospective memory on recognition of item and associative information. Psychology and Aging, 25(2), 486491. Doi: 10.1037/a0017264.

Washburn, A. \& Sands, L. (2006). Social cognition in nursering home residents with and without cognitive impairment. Journal of Gerontology: Psychological Sciences, 61, 174179. Doi: 0.1093/geronb/61.3.P174. 